\title{
ESTABLISHING INDOOR SUBSPACING REQUIREMENTS OF AN LOD (LEVEL OF DETAIL) MODEL FOR GENERATING NETWORK-BASED TOPOLOGICAL DATA
}

\author{
A. R. C. Claridades ${ }^{1,2}$, H.S. Choi ${ }^{3}$, J. Lee ${ }^{1 *}$ \\ ${ }^{1}$ Dept. of Geoinformatics, University of Seoul, Dongdaemun-gu, Seoul, South Korea - \{uosgrad2019012, jlee $\} @$ uos.ac.kr \\ ${ }^{2}$ Dept. of Geodetic Engineering, University of the Philippines Diliman, Quezon City, Philippines \\ ${ }^{3}$ Korea Institute of Civil Engineering and Building Technology, Goyang-si, South Korea - hyunsang@kict.re.kr
}

KEY WORDS: topological data model; subspacing; level of detail, indoor space

\begin{abstract}
:
Nowadays, the complexity of structures in urban environments and the interest in location-based applications increase simultaneously. Along with this is the rise in demand for the firm establishment of data models representing these spaces. Establishing network models that portray topological relationships of space have strengthened support for navigation applications. However, researchers have revisited the limitations of existing standards. As analogous standards have specifications for expressing space at various scales, most have focused on outdoor space or the geometric aspect. Hence, this paper proposes subspacing requirements for a Level of Detail (LOD) model for network-based topological data. We examine various constraints that influence space partition and align these with various application cases for indoor navigation. Through these, we investigate appropriate space subdivision approaches for each level according to applicable constraints and recommended applications. This study poses as an initial study towards establishing a general framework for implementing a 3D hierarchical network-based topological data model.
\end{abstract}

\section{INTRODUCTION}

The spatial data requirements for indoor location-based services (LBS) increase continuously with the accelerated development of urban areas and complex structures. Representation of topological relationships of spaces through network-based data forms the backbone of these applications, especially when focusing on navigation and guidance applications. With the OGC's (Open Geospatial Consortium)'s publication of IndoorGML (OGC (Open Geospatial Consortium), 2018), construction of these types of spatial data has been standardized across a wide range of space sizes and complexities. This document addresses urgent demands for standardization related to indoor LBS, routing, emergency situations, and eventually management of indoor facilities.
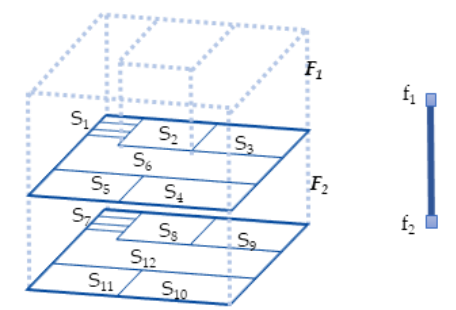

Primal Space

Logical NRS (building level) Logical NRS (corridor level)

Figure 1. Topological relationships in a node hierarchy in NRS

IndoorGML's network structure composed of nodes and edges represents the indoor spaces and their topological relationships, respectively. This standard's theoretical background is based on the Node-Relation Structure (NRS), a logical data model which utilizes the Poincare Duality to convert primal space into dual space (Lee and Kwan, 2005). Notably, the NRS is described as a hierarchical model, where a higher-level node from the network can represent a sub-network composed of lower-level nodes. In
Figure 1, an entire building floor level is abstracted as a single master_node on a higher-level NRS that represents connectivity relationships among floor levels. In turn, this node inherits a subnetwork composed of nodes representing relationships of spatial units within that floor level.

In other analogous standards, such as in CityGML, the concept of level of detail (LoD) has been one of its vital properties contributing to its popularity and usability. It allows for different representations of the same objects through different LOD according to application requirements (OGC (Open Geospatial Consortium), 2021). The standard specifies the categorization of the LOD and specifications for scale description and accuracy requirements. While the descriptions in the documents are indicated as discussion proposals, these allowed further research for refining the LOD requirements and different use-cases (Biljecki et al., 2016).

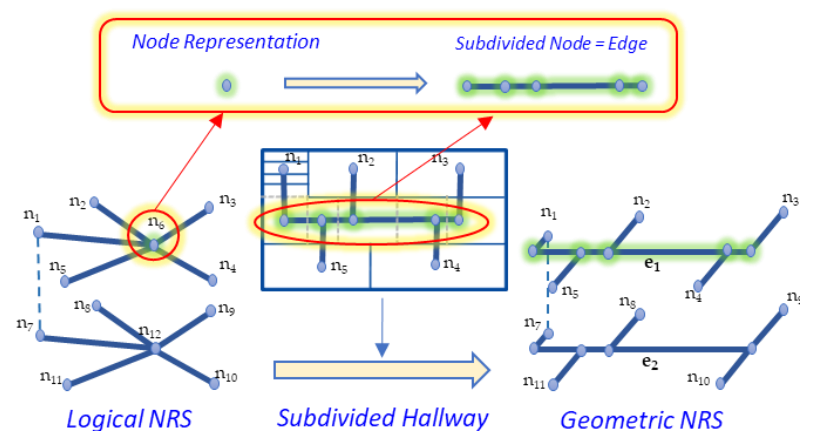

Figure 2. Representation of indoor space relationships using the Geometric NRS

The Logical NRS is the fundamental representation of topological relationships in an indoor environment. However, these relationships are not sufficient to perform network-based

Corresponding author 
analysis that requires geometric information such as shortest-path algorithms. The Geometric NRS is the network model in which edges contain geometric data. In order to determine these properties, such as distance, the manner of how the space is subdivided by the sub-units each node represents must be established. In Figure 2, the hallway spanning the $2^{\text {nd }}$ floor is represented as Node $\mathrm{N}_{6}$. In order to reflect the geometry of the rooms relative to the hallway, the hallway is decomposed into smaller sub-units. In the Geometric NRS, the hallway is represented as the Edge $E_{1}$ and the corresponding nodes lying on this edge. Hence, these edges and nodes in the Geometric NRS are a sub-network of the master_node $\mathrm{N}_{6}$ in the Logical NRS.

Essentially, the hierarchical structure of NRS data enables multiscale representations of the internal configuration of buildings (Lee, 2004). However, while previous studies defined this property, there are no specifications on developing sub-networks down a node hierarchy. In order to be able to perform integrated spatial operations from the macro-level (e.g., room to room navigation) towards the micro-level (e.g., among features within a room), a framework for generating multi-level topological network data is necessary.

As more studies tend to focus on the establishment of indoor data, practical applications are rarely found. Furthermore, identifying applications is required to understand how indoor topological data is used based on different user requirements. In this paper, we propose a level of detail specification for establishing indoor network-based topological data. Based on the current status and published applications for indoor navigation, we identify the constraints that apply to each level. We also identify appropriate spatial subdivision methods corresponding to degrees of resolution. This paper is structured as follows. The next section discusses studies related to specifying LOD specifications, approaches to subspacing, and application cases of indoor navigation. Based on these, we present the proposed LOD framework for indoor network-based topological data. The final section discusses the conclusions of the study and further studies on the topic.

\section{RELATED STUDIES}

The international standard CityGML has introduced the concept of LOD in its specification document with the goal of efficient visualization and analysis of $3 \mathrm{D}$ city models. The simultaneous representation at various scale levels of the same object has given the flexibility to users to select appropriate datasets to use in corresponding applications. While there are different representations across LODs, the lower LODs may be generated by aggregating objects at higher LODs, as implemented in its model (OGC (Open Geospatial Consortium), 2021).

This original specification proposed five levels of detail, from LOD0 to LOD4, primarily focusing on the representation of buildings. A more refined LOD specification was published (Biljecki et al., 2016) to overcome a lack of precise specification and inseparability of each level. While still compliant with the original levels, LOD0 to LOD3 was further subdivided into refined LODs to address ambiguity while still not hindering modeling freedom.

As this CityGML LOD design is intended to apply to any use case of city models, the generic characteristic became a disadvantage since it was not designed to be optimal for a specific case. A context-aware heterogenous LOD (CLOD) standard was proposed by Tang et al. (2020). It introduced a bottom-up approach to building components instead of the building itself (Tang et al., 2020).

However, as the study of human activities happening indoors has become increasingly important, spatial applications for indoor space have been gaining attention and interest. For indoor space, there have been several proposed LOD standards. Indoor space data is classified for construction according to requirements for accuracy and level of detail. Compared to CityGML, where the LODs are defined based on geometrical representation, this specification is classified based on data type and representation. Accordingly, appropriate usage and suitable use cases are presented for each level (Kang and Lee, 2014).

A modified version of Kang and Lee's established LOD is the basis of assigning required LOD for identified critical use cases for indoor space data, together with indoor positioning accuracy specification. Results show that use cases relevant for public administration services require only low LODs and can guide government agencies regarding acquisition and management of spatial data and future efforts for its establishment and provision (Park and Lee, 2017).

Recent researches addressed the limitations of CityGML LOD4 for indoor space by expanding the indoor LOD (ILOD) specification. Through the advantages of IndoorGML and IFC, semantic aspects of structure, connectivity, and volume became the basis of defining more detailed levels. Through a combination of the CityGML LODs and ILOD, a Full LOD (FLOD) is also proposed to realize full specification for $3 \mathrm{D}$ building models (Tang et al., 2018).

However, these LOD specifications of spatial data are for geometry data or visualization purposes. While these aspects of spatial data may greatly benefit from this concept, LODs for network-based topological data are still an unexplored area. Lee (2005) illustrated the concept of master_node through a node representing a hallway, decomposed into a set of nodes and edges. This process of decomposition may be developed further for a more detailed network representation in indoor space.

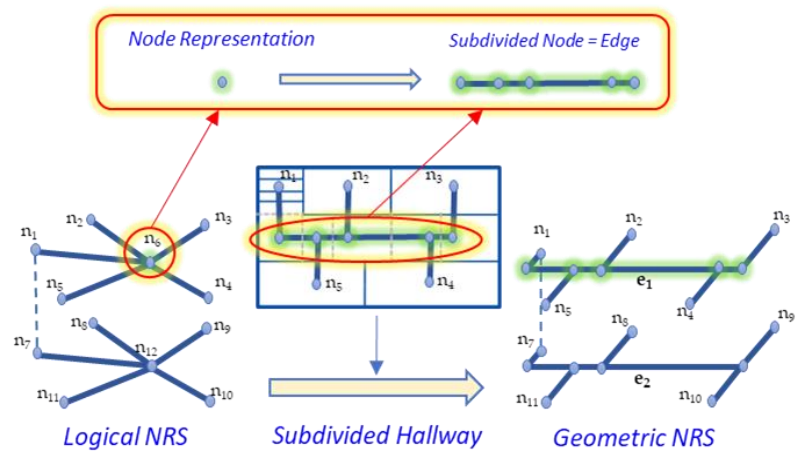

Figure 3. Generating lower-level (more detailed) Geometric NRS through space subdivision

Consider a node that represents a room in a corridor-level Geometric NRS in Figure 3. If a lower-level network is generated, it will correspond to a network representing topological relationships of sub-units within the room. Therefore, we must determine how this room, already a sub-unit of the whole floor level, is decomposed into further sub-units in order to generate the room level sub-network comprising the master_node in the corridor level network. The IndoorGML 
standard refers to this process of decomposing indoor space as subspacing.

The subspacing process allows the expression of hierarchical structures of indoor space through a space subdivision process, where a sub-unit of indoor space is divided to reflect geometric properties (OGC (Open Geospatial Consortium), 2018). Furthermore, this process allows the generation of more detailed network representations to reduce inefficiency in route planning, more accurate indoor localization (Diakité and Zlatanova, 2018). Therefore, a subspacing methodology must be defined in order to completely generate a multi-level topological representation of indoor space to portray its hierarchical properties fully.

Geometric properties have primarily dictated subdivision of spaces, and in most 2D approaches, semantics is not elaborately considered. In indoor positioning, space subdivision is used in the context of the efficient deployment of sensors. The locations of sensors within the space must be efficiently decided to save cost while maintaining high accuracy. Sensor deploymentoriented subspacing has primarily used Thiessen Polygons (Aurenhammer, 1991; Lin et al., 2013) or equal grids calculated from sensor coverage (Bulusu et al., 2001). On the other hand, indoor paths have also determined how sub-units of space are derived. Besides geometric properties, semantic information is an additional basis of the partitioning of space, and for which partitions require further subspacing (Diakité and Zlatanova, 2018).

Various methods of subspacing are demonstrated in previous studies. These partitions are used in a variety of context, particularly for positioning and navigation in indoor space (Zlatanova et al., 2020). Lee (2004) followed up the NRS with a complementary geometric model, named the Geometric Network Model (GNM), to more accurately portray the indoor layout (Lee, 2004). In the GNM, the corridor was more realistically portrayed as a set of nodes and edges instead of a single node, as shown previously in Figure 2. This was achieved through the Straight Median Axis Transform (SMAT), a partial subdivision method applied on a convex-shaped hallway.

However, due to computational limitations, SMAT is not optimal for non-convex-shaped partitions. In these cases, free spaces must be determined adequately since the non-convex spaces are more varied in shape or structure. Partial subdivision methods suitable for irregular shapes, such as Visibility Graphs (Hwang, 2013), can achieve finer subspaces compared to SMAT, and resulting subspaces still differ in size, depending on the layout of the navigable spaces to be connected.

Furthermore, some large subspaces are subdivided differently compared to smaller ones, thus resulting in more unsubspaced partitions. Because remaining free spaces in indoor space are more varied in shape and structure, complete subdivision methods are suitable. Such methods, such as Vonoroi diagrams, Convex Cell Optimization (Lamarche and Donikian, 2004), and Z-Buffer Subdivision (Geraerts, 2010), allow for considering whitespaces within the target spaces, but still, result in differently-shaped and sized partitions.

Full subspacing that results in uniform partitions is achieved through a process of tessellation. This paradigm considers indoor spaces as continuous spaces and is subdivided into regularly sized cells or grids so that no partitions remain unsubdivided. While the cells do not represent rooms or physical divisions, they represent occupancies, the granularity of which is based on the cell size, which also corresponds to edge distance (Li et al., 2010).

\section{SUBSPACING FRAMEWORK FOR NETWORK TOPOLOGICAL DATA}

In this study, we propose an indoor space LOD specification for subspacing in order to generate a network-based topological representation of indoor space. We identify the factors that influence the decomposition of indoor spaces relative to navigable spaces in order to generate the node-relation structure. We classify which constraints determine the structure of the NRS for each scale, hence dictating the method to generate networks at appropriate levels.

\subsection{Indoor Space Constraints}

Navigation in indoor space is primarily influenced by constraints such as the layout of the rooms, corridors, as well as the presence and absence of obstacles and access points. Consequently, these constraints differ depending on the scale within the indoor space. For example, within a floor level, navigation paths are influenced by the arrangement of the rooms along a hallway, but within a room, routes can be drawn according to position and number of doors and the arrangement of free space unoccupied by facilities.

From the perspective of navigation, indoor spaces can be classified according to their functions. These functional constraints categorize locations into either a place where people stay, such as rooms, or transfer spaces that facilitate movement between these spaces. Stay spaces are often the targets of navigation and where human activities are conducted. On the other hand, Transfer spaces connect the stay spaces such as hallways and staircases (Kang et al., 2015).

However, some of the stay spaces, such as auditoriums, lobbies or halls, may be subspaced further as its large extent would mean a single node representation is insufficient (Kang et al., 2015). Some smaller-sized rooms may also have multiple entry points, hence having multiple possible navigation paths. These are semantic constraints that must be considered in generating a more detailed navigation network.

Furthermore, facility constraints must also be considered since navigable spaces also contain facilities that reduce the available free spaces that can actually be used for navigation. Apart from the spaces that these objects actually occupy, the spaces needed during the use of these facilities also influence the determination of free spaces and influence how space is eventually partitioned (Diakité and Zlatanova, 2018). Finally, the characteristics of agents, such as their size and locomotion types, also influence subspacing in the context of determining navigation paths. These types of constraints are also set to be considered in the next version of IndoorGML (Diakité et al., 2020).

\subsection{Indoor Space Navigation across Different Scales}

Navigation in indoor space can be viewed at different scale levels. A building is differentiated from outdoor spaces by the presence of stories at different elevation values. Hence, the initial level of navigation is the movement across floor levels. This level of navigation is sufficient in applications that depend only on vertical movement across floors, the differences in elevation, or in structures that have simple layouts. This is also suitable for applications where target spaces of navigation are on a single floors- such as basements, helicopter landing pads, or rooftops. 
Intuitively, navigation may occur within a floor level. Most applications illustrating indoor network models are at this level. This is particularly useful for emergency evacuation applications where building occupants can spontaneously start their cognitive path calculation at the door of their current room location and will only have to need path guidance at the hallways. In this level, the hallways must be partitioned to reflect the layout of the rooms within the floor level. However, a single room, regardless of size, is regarded as a single location in the network.

However, in more detailed path planning and guidance applications, especially in the context of emergency navigation, rooms must be differentiated based on size, layout, or importance. For example, large rooms may have more than one exit point. Hence they may contain multiple paths towards the exit. Lobbies may contain multiple possible paths too, and halls may contain smaller rooms within that may affect how paths are determined.

Objects within indoor space also present a vital constraint because they affect the actual free spaces within the rooms. These objects can play a role in how the paths may be modified. For example, in auditoriums, the manner in how the seats are arranged dictates the paths of the occupants. Large furniture can act as obstacles or even may create smaller rooms within a room.

Finally, indoor navigation can be viewed in its finest possible detail when space is fully subdivided into grids. This is applicable for agent-based simulation of navigation or in the navigation of non-human agents such as drones, robots. Here, agents are not constrained by linear paths since the spaces are treated as continuous.

\subsection{Proposed LOD Specification}

As discussed in the previous sub-section, indoor space and, correspondingly, navigation within can be viewed at various levels of scale. Representing indoor network navigation data in this manner allows for multiple but simultaneous representations for multi-resolution visualization and efficient analysis. In the context of possible application use cases at various levels, Figure 4 illustrates the five levels of detail for representing indoor navigation networks. The coarsest level is at Level 0 , where each node represents a floor level of a structure, each differentiated only by a height value. Nodes at this level are illustrated as a master_node in the CDM (Lee and Kwan, 2005). Also shown in the CDM, and eventually, in the GNM, Level 1 contains navigations across sub-units (rooms) along the hallways at an individual floor level. Meanwhile, Level 2 networks consider further subdivision across large rooms, lobbies, or halls. At Level 3 , navigation paths reflect the actual free spaces within the rooms remaining after considering objects in indoor space, and Level 4 is the finest scale where navigable spaces are divided as cells.

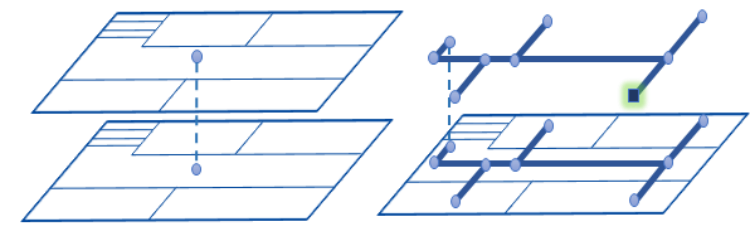

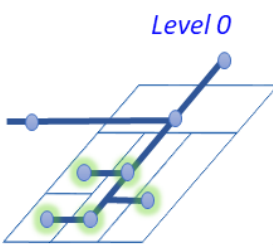

Level 2

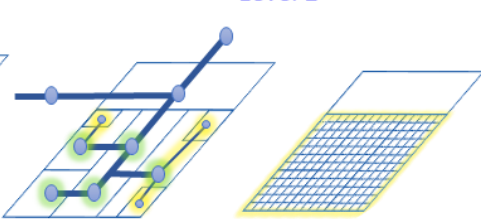

Level 3

Level 4

Figure 4. The Five Levels of detail for representing indoor navigation networks

Table 1 summarizes the proposed specifications for subdividing indoor space in order to generate the desired LOD for the network data. The number of applicable constraints increases along with the hierarchical levels, along with the complexity of the preferred subdivision method. Naturally, since a sub-network in the lowerlevel hierarchy (for example, Level 2) inherits from a higherlevel network (such as Level 1) node, the resulting subspaces are smaller in size.

At Level 1, functional constraints dictate the differentiation of spaces where agents stay (such as rooms) versus spaces they use as transitions from one stay space to another (hallways). A partial subdivision is suitable for a convex-shaped polygon, like the hallway that must be subdivided.

At Level 2, semantic constraints must be applied in order to determine the utilization of the space, which dictates if it is necessary to be further subdivided. Some of these spaces may also have more general shapes, so partial subspacing methods for irregular polygons are suitable.

\begin{tabular}{|cllll|}
\hline & \multicolumn{1}{c}{ Level 1 } & \multicolumn{1}{c|}{ Level 2 } & \multicolumn{1}{c|}{ Level 3 } & \multicolumn{1}{c|}{ Level 4} \\
\hline $\begin{array}{c}\text { Applicable } \\
\text { Constraints }\end{array}$ & Functional & $\begin{array}{l}\text { Functional } \\
\text { Semantic }\end{array}$ & $\begin{array}{l}\text { Functional } \\
\text { Semantic } \\
\text { Facilities }\end{array}$ & $\begin{array}{l}\text { Functional } \\
\text { Semantic } \\
\text { Facilities } \\
\text { Agents }\end{array}$ \\
\hline $\begin{array}{c}\text { Space Subdivision } \\
\text { Method }\end{array}$ & $\begin{array}{l}\text { Partial Subdivision - } \\
\text { Convex Polygon }\end{array}$ & $\begin{array}{l}\text { Partial Subdivision }- \\
\text { Irregular Polygon }\end{array}$ & $\begin{array}{l}\text { Complete Irregular } \\
\text { Subdivision }\end{array}$ & $\begin{array}{l}\text { Complete Regular } \\
\text { Subdivision }\end{array}$ \\
\hline $\begin{array}{c}\text { Subspace Size } \\
\text { Coarse }\end{array}$ & Fine & Finer & Finest \\
\hline Applications & Corridor-level, room to \\
& room navigation & $\begin{array}{l}\text { Navigation within large } \\
\text { rooms (spaces with } \\
\text { multiple entrances, } \\
\text { halls, lobbies) }\end{array}$ & $\begin{array}{l}\text { Sub-room level } \\
\text { navigation; } \\
\text { Considering fixed } \\
\text { indoor facilities }\end{array}$ & $\begin{array}{l}\text { Robot navigation; } \\
\text { Considering movable } \\
\text { indoor facilities, } \\
\text { dynamic agents }\end{array}$ \\
\hline
\end{tabular}

Table 1. Specifications for applicable constraints and subspacing methods for each LOD 
Level 3 networks consider facility constraints as an additional factor since immovable and semi-movable objects must be regarded. The spaces occupied by these objects must be removed prior to subspacing, resulting in a more structurally varied remaining space. Hence a complete subdivision method is suitable at this level.

Finally, Level 4 networks consider agent constraints. Subdivision parameters are based on factors including size (occupied space) and locomotion method (walking, flying, or dispersed, in the case of environmental agents such as smoke). Agents may travel continuously in space on the remainder of the object spaces, subdivided equally into grids or cells.

\section{CONCLUSION}

In order to provide relevant and accurate services, LBS applications must be built on spatial data that represents the real world realistically. Indoor space can be viewed at various scales from the perspective of navigation. While analogous data models such as CityGML have specifications for multi-scale data representation, and the same has been explored for indoor geometric datasets, literature has been lacking in describing Levels of detail for topological data, especially network-based models.

In this study, we presented an indoor space LOD specification for network-based topological data. By investigating present constraints in indoor space and aligning with applications for navigation at different scales, we identify corresponding space partitioning techniques that may be suitable for each level.

This paper is an initial study towards addressing the gap in the literature on how to generate complete multi-level network data representing spatial relationships. These spatial relationships include topological relationships between the connected and adjacent spaces and, more importantly, represent hierarchical relationships between a space and the sub-units of space that compose it. While this paper only covers the specifications for each LOD and how to generate the sub-units through subspacing, it is a prerequisite to defining the complete hierarchical topological data model.

\section{ACKNOWLEDGEMENTS}

This research was supported by a grant (21NSIP-B135746-05) from National Spatial Information Research Program (NSIP) funded by Ministry of Land, Infrastructure and Transport of Korean government and the National Research Foundation of Korea (NRF) grant funded by the Korea government (MSIT) (No. 2021R1A2C1013951).

\section{REFERENCES}

Aurenhammer, F., 1991. Voronoi Diagrams - a Survey of a Fundamental Geometric Data Structure. ACM Comput. Surv. 23, 345-405. doi.org/10.1145/116873.116880

Biljecki, F., Ledoux, H., Stoter, J., 2016. An improved LOD specification for 3D building models. Comput. Environ. Urban Syst. 59, 25-37. doi.org/10.1016/j.compenvurbsys.2016.04.005

Bulusu, N., Heidemann, J., Estrin, D., 2001. Adaptive Beacon Placement, in: Proceedings of the 21st International Conference on Distributed Computing Systems \{(ICDCS\} 2001), Phoenix, Arizona, USA, April 16-19, 2001. \{IEEE\} Computer Society.

Diakité, A.A., Zlatanova, S., 2018. Spatial subdivision of complex indoor environments for 3D indoor navigation. Int. J. Geogr. Inf. Sci. $213-235$. doi.org/10.1080/13658816.2017.1376066

Diakité, A.A., Zlatanova, S., Alattas, A.F.M., Li, K.J., 2020. Towards IndoorGML 2.0: Updates and Case Study Illustrations. ISPRS - Int. Arch. Photogramm. Remote Sens. Spat. Inf. Sci. XLIII-B4-2, 337-344. doi.org/10.5194/isprs-archives-XLIII-B42020-337-2020

Geraerts, R., 2010. Planning short paths with clearance using explicit corridors. Proc. - IEEE Int. Conf. Robot. Autom. 19972004. doi.org/10.1109/ROBOT.2010.5509263

Hwang, Y., 2013. Network communities in the visibility graph: A new method for the discretization of space. 2013 Int. Sp. Syntax Symp.

Kang, H.Y., Jung, H., Lee, J., 2015. A Study of Subspacing Strategy for Service Applications in Indoor Space. J. Korea Spat. Inf. Soc. 23, 113-122.

Kang, H.Y., Lee, J., 2014. A Study on the LOD (level of detail) Model for Applications Based on Indoor Space Ddata. J. Korean Soc. Surv. Geod. Photogramm. Cartogr. 32, 143-151. doi.org/10.7848/ksgpc.2014.32.2.143

Lamarche, F., Donikian, S., 2004. Crowd of virtual humans: A new approach for real time navigation in complex and structured environments. Comput. Graph. Forum 23, 509-518. doi.org/10.1111/j.1467-8659.2004.00782.x

Lee, J., 2004. A Spatial Access-Oriented Implementation of a 3D GIS Topological Data Model for Urban Entities. Geoinformatica 15, 1-7.

Lee, J., Kwan, M.P., 2005. A Combinatorial Data Model for Representing Topological Relations among 3D Geographical Features in Micro-Spatial Environment. Int. J. Geogr. Inf. Sci. 19, 1039-1056. doi.org/10.1080/13658810500399043

Li, X., Claramunt, C., Ray, C., 2010. A grid graph-based model for the analysis of 2D indoor spaces. Comput. Environ. Urban Syst. $\quad 34, \quad$ 532-540. doi.org/10.1016/j.compenvurbsys.2010.07.006

Lin, Z., Zhang, S., Yan, G., 2013. An incremental deployment algorithm for wireless sensor networks using one or multiple autonomous agents. Ad Hoc Networks 11, 355-367. doi.org/https://doi.org/10.1016/j.adhoc.2012.06.009

OGC (Open Geospatial Consortium), 2021. CityGML v.3.0.0.

OGC (Open Geospatial Consortium), 2018. IndoorGML v.1.0.3. URL http://docs.opengeospatial.org/is/14-005r4/14-005r4.html

Park, J., Lee, J., 2017. Establishing Required LOD And Positioning Accuracy for Indoor Spatial Information Applications in Public Administrative Works. J. Korean Soc. Surv. Geod. Photogramm. Cartogr. 35, 103-112. doi.org/10.7848/ksgpc.2017.35.2.103 
Tang, L., Li, L., Ying, S., Lei, Y., 2018. A full level-of-detail specification for 3D building models combining indoor and outdoor scenes. ISPRS Int. J. Geo-Information 7. doi.org/10.3390/ijgi7110419

Tang, L., Ying, S., Li, L., Biljecki, F., Zhu, H., Zhu, Y., Yang, F., Su, F., 2020. An application-driven LOD modeling paradigm for 3D building models. ISPRS J. Photogramm. Remote Sens. 161, 194-207. doi.org/10.1016/j.isprsjprs.2020.01.019

Zlatanova, S., Yan, J., Wang, Y., Diakité, A.A., Isikdag, U., Sithole, G., Barton, J., 2020. Spaces in spatial science and urban applications-state of the art review. ISPRS Int. J. GeoInformation 9, 1-28. doi.org/10.3390/ijgi9010058 